This is a self-archived version of an original article. This version may differ from the original in pagination and typographic details.

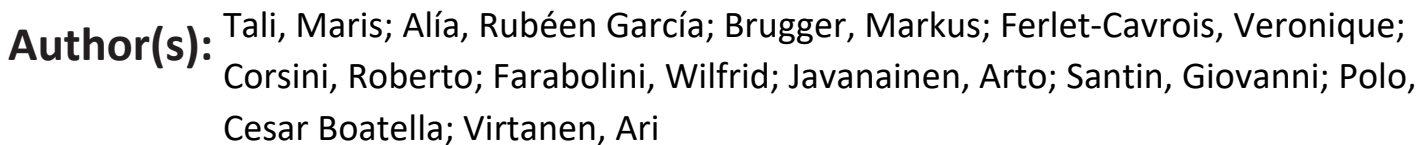

Title: Mechanisms of Electron-Induced Single Event Latchup

Year: 2019

Version: Accepted version (Final draft)

Copyright: @ 2019 IEEE

Rights: In Copyright

Rights url: http://rightsstatements.org/page//nC/1.0/?language=en

Please cite the original version:

Tali, M., Alía, R. G., Brugger, M., Ferlet-Cavrois, V., Corsini, R., Farabolini, W., Javanainen, A., Santin, G., Polo, C. B., \& Virtanen, A. (2019). Mechanisms of Electron-Induced Single Event Latchup. IEEE Transactions on Nuclear Science, 66(1), 437-443.

https://doi.org/10.1109/TNS.2018.2884537 


\title{
Mechanisms of Electron-Induced Single-Event Latchup
}

\author{
Maris Tali , Rubén García Alía , Markus Brugger, Véronique Ferlet-Cavrois, Roberto Corsini, \\ Wilfrid Farabolini, Arto Javanainen , Giovanni Santin , Cesar Boatella Polo, and Ari Virtanen
}

\begin{abstract}
In this paper, possible mechanisms by which electrons can induce single-event latchups in electronics are discussed. The energy deposition and the nuclear fragments created by electrons in silicon are analyzed in this context. The cross section enhancement effect in the presence of high- $\mathrm{Z}$ materials is discussed. First experimental results of electron-induced latchups are shown in static random access memory devices with low linear energy transfer thresholds. The radiation hardness assurance implications and future work are discussed.
\end{abstract}

Index Terms-Electrons, static random access memory (SRAM).

\section{INTRODUCTION}

$\mathbf{E}$ NERGETIC particles in space cause various failures in electronics. One of the more dangerous among these is the single-event latchup (SEL), because the only way to remove the potentially destructive effect is to power cycle the device. A large body of work exists describing the approaches behind and radiation hardness assurance (RHA) qualification for SEL induced by protons [1]-[3] and neutrons [4]. Likewise, work is available showing electrons are capable of producing soft errors [5]-[7], however, no experimental evidence of hard failures is so far available. The discussion around the electroninduced radiation effects in electronics has been motivated by the JUpiter ICy moon Explorer mission (JUICE). In the Jovian environment, electrons can reach energies up to a few hundred megaelectronvolts and are the main contributor to the total absorbed doses received by electronics [8].

It has been shown in [6] through simulations and experimental results that electrons and photons can produce soft errors through high-linear energy transfer (LET) via nuclear interactions products with silicon. Such fragments are similar to those produced by protons [9] and can reach LET (in $\mathrm{Si}$ ) values of $13 \mathrm{MeVcm}^{2} / \mathrm{mg}$ for electrons and $15 \mathrm{MeVcm}^{2} / \mathrm{mg}$

Manuscript received July 12, 2018; revised October 16, 2018 and November 21, 2018; accepted November 25, 2018. Date of current version January 17, 2019. This work was supported in part by CERN through CERN/ESA Collaboration, VESPER, CHARM, and R2E Groups and in part by the European Space Agency, ESTEC under Contract 4000115379/15/NL/MH/GM.

M. Tali, A. Javanainen, and A. Virtanen are with the Department of Physics, University of Jyväskylä, 40014 Jyväskylä, Finland (e-mail: maris.tali@cern.ch).

R. G. Alía, M. Brugger, R. Corsini, and W. Farabolini are with CERN, 1211 Geneva, Switzerland.

V. Ferlet-Cavrois, G. Santin, and C. Boatella Polo are with ESA/ESTEC, 2201 AZ Noordwijk, The Netherlands

Color versions of one or more of the figures in this paper are available online at http://ieeexplore.ieee.org.

Digital Object Identifier 10.1109/TNS.2018.2884537 for photons, however, with a much lower probability than that associated with proton and nuclear reactions [6]. Therefore, it is expected that these recoils are in principle also capable of inducing hard errors in devices with a low enough LET threshold. Such effects include SELs or even single event burnouts.

Through experiments at the high-energy (HE) electron linear accelerator (LINAC) test facility VESPER at CERN, first SEL events were observed in static random access memories (SRAMs). Three different memories were tested with varying LET thresholds. The memories are highly sensitive to SEL effects, with an LET threshold of below $\sim 3 \mathrm{MeVcm}^{2} / \mathrm{mg}$. It was expected that the LET production distribution of electrons and photons could also generate SEL for a low enough LET threshold. The experimental validation confirms this, as the associated energy, range, and angular distribution of the nuclear fragments are compatible with SEL induction.

It has been shown previously that high- $Z$ materials present in some ICs greatly increases the probability of causing proton-induced latchup events [2]. In addition, this phenomenon introduces a very strong cross section dependence with energy, therefore, requiting the need for testing at the maximum energy present in the working environment. In this paper, energy deposition simulations were made in FLUKA [10], [11] for electrons, using an integral rectangular parallelpiped (IRPP) geometry previously described in [12]-[14]. The geometry includes a layer of tungsten to show the effect of the electron-tungsten interaction recoils on the overall energy deposition cross section. An estimated cross section was found by folding the experimental heavy-ion cross section, measured in earlier work [12], and the FLUKA energy deposition simulations.

In addition, a simulation was performed to characterize the nuclear reaction products created by photons and electrons in tungsten as an extension for work done for protons in [2]. This paper shows for the first time that photons and electrons are also capable of inducing fissions fragments with LETs as high as $\sim 40 \mathrm{MeVcm}^{2} / \mathrm{mg}$.

\section{FACILITy AND TESt SETUP}

The experiments were conducted at the VESPER test bench located at the CLEAR experimental area at CERN [6]. The CLEAR facility is a general purpose electron LINAC for electronics, materials testing, and accelerator technology development with energies ranging from 60 to $200 \mathrm{MeV}$. 


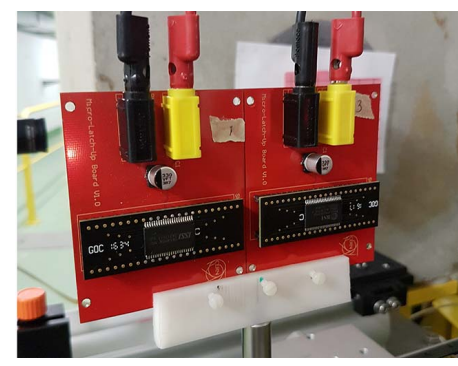

Fig. 1. Experimental setup for the SEL experiment at VESPER, test boards with SRAMs. The SRAM were moved into the beam by a movable stage.

TABLE I

Summary of the SRAM Memories Tested at VESPER [12]

\begin{tabular}{cccc}
\hline Shorthand & Version & Size & Tech Node \\
\hline Brilliance & BS62LV8001BIP55 & $8 \mathrm{Mbit}$ & $180 \mathrm{~nm}$ \\
ISSI & IS61LV5128AL-10TLI & $4 \mathrm{Mbit}$ & $180 \mathrm{~nm}$ \\
Alliance & AS7C34098A-10TCN & $4 \mathrm{Mbit}$ & $200 \mathrm{~nm}$ \\
\hline
\end{tabular}

The beam that was used is a dark current beam with a low charge from 3.5 to $20 \mathrm{pC}$ per pulse. The beam was diffused using a chromox screen several meters upstream of the test position to cover the SRAM device on the test board.

The test board was attached to the movable table which was moved in the beam for testing. The beam size and intensity were logged by a fast beam current transformer located just in front of the device under test (DUT) and by a sensitive yttrium aluminum garnet fluorescent screen. Both of the detectors are logged and the variation of the beam size and intensity is saved during the test.

The devices were biased to $3.3 \mathrm{~V}$, which is the nominal working current. The labview system controls the Agilent power supply and supervises the latchup system and performs the delatching. For all the runs, the dark current beam was used at VESPER [6]. The measurements were performed at room temperature

\section{A. Devices Under Test}

The DUTs were three SRAM memories from three different manufacturers (ISSI, Brilliance, and Alliance), as shown in Fig. 1. A summary of the three memories can be seen in Table I. The Brilliance device has been characterized by both a heavy-ion beam and proton beams [12], [14], where they were referred to as SRAM F and BS62, respectively. The devices were biased through a programmable Agilent power supply, which was also used for delatching purposes. The SEL detection limit was set to $100 \mathrm{~mA}$ and the current was limited to $200 \mathrm{~mA}$ during the latchup events. The hold time was $300 \mathrm{~ms}$ and the cut time was $2 \mathrm{~s}$. The total standby current consumption of the DUT differed per memory and was $30 \mathrm{~mA}$ for the ISSI, $10 \mu \mathrm{A}$ for the Brilliance (the memory was in the low-power shut down mode during testing) and $12 \mathrm{~mA}$ for the Alliance memory.

The Brilliance SRAM used in this paper has a heavyion LET threshold $2.4 \mathrm{MeV} \mathrm{cm}^{2} / \mathrm{mg}$. The date code for these parts is 09254 , which is equivalent to the SRAM F described
TABLE II

Summary of the Weibull Parameters of the SRAM Memories Tested at VESPER, ISSI, AND Alliance From [12], BRILliance From [14]. *The Lo OF 0 FOR the Alliance Device Is UNPHYSICAL AND SHOUlD BE CONSIDERED AS $<5 \mathrm{MeVcm}^{2} / \mathrm{mg}$, Which THE LOWest LET VALue For WHICH EVENTS WERE OBSERVED [15]

\begin{tabular}{cccccc}
\hline Shorthand & $\begin{array}{c}\text { XSsat } \\
\left(\mathbf{c m}^{2}\right)\end{array}$ & $\begin{array}{c}\text { Lo } \\
(\mathbf{M e V} \\
\left.\mathbf{c m}^{2} / \mathbf{m g}\right)\end{array}$ & $\begin{array}{c}\mathbf{L}_{1 \%} \\
(\mathbf{M e V} \\
\left.\mathbf{c m}^{2} / \mathbf{m g}\right)\end{array}$ & $\begin{array}{c}\mathbf{W} \\
(\mathbf{M e V} \\
\left.\mathbf{c m}^{2} / \mathbf{m g}\right)\end{array}$ & $\mathbf{s}$ \\
\hline ISSI & 0.24 & 2.1 & 6.5 & 16.8 & 3.4 \\
Alliance & 0.13 & $0 *(<5)$ & 15 & 27.6 & 7.4 \\
Brilliance & 0.60 & 2.4 & 3.7 & 13.7 & 1.8 \\
\hline
\end{tabular}

in [12]. The Alliance memory is referred to in the same work as SRAM C, with a threshold of $<5 \mathrm{MeVcm}^{2} / \mathrm{mg}$ [15], and the ISSI memory as SRAM A, with a threshold of $2.1 \mathrm{MeV} \mathrm{cm}^{2} / \mathrm{mg}$. Recoils generated by electro nuclear and photonuclear reactions in silicon reach LET values of up to $13 \mathrm{MeV} \mathrm{cm}^{2} / \mathrm{mg}$ for electrons and $15 \mathrm{MeV} \mathrm{cm}^{2} / \mathrm{mg}$ for photons, as discussed further in Section IV-C and shown in [6]. This means that the main contribution to the SEL events observed in this SRAM during irradiation can be attributed to high-LET recoils created in the silicon. Since this SRAM has a high SEL sensitivity, it is a good first experimental proof of electron-induced SEL. In addition to the LET threshold, a more important value in terms of the cross section is the $L_{1 \%}$ value, which is the so-called onset LET value or the value at which the cross section is $1 \%$ of the saturation cross section [16]. The value is $15 \mathrm{MeVcm}^{2} / \mathrm{mg}$ for the Alliance memory [12], $6.5 \mathrm{MeV} \mathrm{cm}^{2} / \mathrm{mg}$ for the ISSI memory [14] and $3.7 \mathrm{MeV} \mathrm{cm}^{2} / \mathrm{mg}$ for the Brilliance. These values can also be seen in Table II.

\section{EXPERIMENTAL RESULTS}

\section{A. Brilliance Experimental Results}

The ISSI memory has a LET threshold of $2.4 \mathrm{MeV} \mathrm{cm}^{2} / \mathrm{mg}$, and therefore, the cross section is dominated by recoils generated by electronuclear and photonuclear reactions in silicon. The cross section has a visible energy dependence already with the results at $210 \mathrm{MeV}$ having a factor of $\sim 2$ higher cross section than the $160 \mathrm{MeV}$ results. A higher bound is given for the measurement at $60 \mathrm{MeV}$. The experimental data can be seen in Table III. Of the three memories tested, the Brilliance memory has the highest saturation cross section. As predicted by the Weibull fit for heavy ions, the Brilliance memory was also the most sensitive to electron-induced latchups. The cross section at $215 \mathrm{MeV}$ is $1.34 \times 10^{-10}$, which is a $\sim$ three times higher than for the ISSI memory.

As discussed in Section III-D, the limiting factor for the latchup testing is the increase in the standby current in the device. After a certain dose level, the standby current increases and the result after this point are ignored for the cross section calculation.

\section{B. ISSI Experimental Results}

Similar to the Brilliance memory, the ISSI memory has a LET threshold of $2.1 \mathrm{MeV} \mathrm{cm}^{2} / \mathrm{mg}$, and therefore, the cross 
TABLE III

BRILLIANCE SRAM MEMORY IRRADIATION RESULTS AT VESPER

\begin{tabular}{|c|c|c|c|c|c|c|}
\hline $\begin{array}{l}\text { Energy } \\
(\mathrm{MeV})\end{array}$ & $\begin{array}{l}\text { Beam } \\
\text { Charge } \\
\text { (pC) }\end{array}$ & $\begin{array}{l}\text { Beam } \\
\text { size } \mathbf{x} \\
(\mathbf{m m})\end{array}$ & $\begin{array}{l}\text { Beam } \\
\text { size y } \\
(\mathbf{m m})\end{array}$ & $\begin{array}{l}\text { Total } \\
\text { time } \\
\text { (min) }\end{array}$ & $\begin{array}{l}\text { Recorded } \\
\text { SELs }\end{array}$ & $\begin{array}{r}\text { cross-section } \\
\left(\mathrm{cm}^{2} / \text { Device }\right)\end{array}$ \\
\hline 160 & 11 & 12 & 13 & 25 & 26 & $6.14 \times 10^{-11}$ \\
\hline 215 & 11 & 15 & 12 & 360 & 84 & $1.34 \times 10^{-10}$ \\
\hline
\end{tabular}

TABLE IV

ISSI SRAM MEMORY IRRADIATION RESUlTS AT VESPER, *AN UPPER BOUND HAS BEEN GIVEN FOR THE 60-MEV IRRADIATION

\begin{tabular}{|c|c|c|c|c|c|c|}
\hline $\begin{array}{l}\text { Energy } \\
(\mathrm{MeV})\end{array}$ & $\begin{array}{l}\text { Beam } \\
\text { Charge } \\
\text { (pC) }\end{array}$ & $\begin{array}{l}\text { Beam } \\
\text { size } x \\
(\mathbf{m m})\end{array}$ & $\begin{array}{l}\text { Beam } \\
\text { size y } \\
(\mathrm{mm})\end{array}$ & $\begin{array}{l}\text { Total } \\
\text { time } \\
\text { (min) }\end{array}$ & $\begin{array}{l}\text { Recorded } \\
\text { SELs }\end{array}$ & $\begin{array}{l}\text { cross-section } \\
\left(\mathrm{cm}^{2} / \text { Device }\right)\end{array}$ \\
\hline 60 & 12 & 24 & 15 & 31 & 0 & $2.91 \times 10^{-12 *}$ \\
\hline 155 & 11 & 19 & 20 & 51 & 8 & $1.42 \times 10^{-11}$ \\
\hline 160 & 12 & 13 & 13 & 30 & 5 & $9.84 \times 10^{-12}$ \\
\hline 210 & 3.5 & 15 & 24 & 105 & 13 & $3.20 \times 10^{-11}$ \\
\hline 210 & 4 & 15 & 24 & 50 & 6 & $2.72 \times 10^{-11}$ \\
\hline 210 & 4 & 15 & 24 & 27 & 4 & $3.35 \times 10^{-11}$ \\
\hline 215 & 15 & 12 & 13 & 196 & 6 & $1.76 \times 10^{-11}$ \\
\hline
\end{tabular}

section is dominated by recoils generated by electronuclear and photonuclear reactions in silicon. The ISSI memory has a heavy-ion saturation cross section about an order of magnitude lower than the Brilliance memory which is also seen in the electron-induced SEL cross section. The cross section has a visible energy dependence already with the results at $210 \mathrm{MeV}$ having a factor of $\sim 2$ higher cross section than the $160 \mathrm{MeV}$ results. Due to the low SEL counts, the uncertainties of the results are large and more tests at lower energies would be needed to confirm the energy dependence at lower energies. The experimental data can be seen in Table IV.

To assess the pile-up effect, for the ISSI memory a measurement has been made at $210 \mathrm{MeV}$ with a beam charge of $3.5 \mathrm{pC}$ with a cross section of $3.20 \pm 0.88 \times 10^{-11} \mathrm{~cm}^{2} /$ bit and at $215 \mathrm{MeV}$ at $15 \mathrm{pC}$ with a cross section of $1.76 \pm$ $0.72 \times 10^{-11} \mathrm{~cm}^{2} /$ bit. The difference between the cross section is compatible with the experimental uncertainty. The change in cross section is then much less compared to the change in beam charge, which is increased by $4.3 \times$. This result excludes the pile-up effect which would result in an exponential sensitivity of the SEL rate to the pulse charge [5].

\section{Alliance Experimental Results}

The Alliance memory Weibull fit has a $L_{1} \%$ value of $15 \mathrm{MeV} \mathrm{cm}^{2} / \mathrm{mg}$ [13]. This is higher than the LET of the highest silicon recoils created by electrons of $13 \mathrm{MeV} \mathrm{cm} / \mathrm{mg}$. Therefore, as is the case for protons, the SEL cross section for this component is expected to be dominated by tungsten fission fragments, which are produced in a much smaller proportion than silicon fragments owing to the smaller associated volume.

Therefore, no latchup events were observed prior to the standby current consumption becoming higher than the selected SEL threshold level of $200 \mathrm{~mA}$. All of the results
TABLE V

Alliance SRAM Memory IrRadiation Results at VESPER, *an UPPER BOUND HAS BEEN GIVEN FOR THE IRRADIATIONS AS NO SEL WAS OBSERVED DURING THE TESTS

\begin{tabular}{ccccccc}
\hline $\begin{array}{l}\text { Energy } \\
(\mathbf{M e V})\end{array}$ & $\begin{array}{l}\text { Beam } \\
\text { Charge } \\
(\mathbf{p C})\end{array}$ & $\begin{array}{l}\text { Beam } \\
\text { size x } \\
(\mathbf{m m})\end{array}$ & $\begin{array}{l}\text { Beam } \\
\text { size y } \\
(\mathbf{m m})\end{array}$ & $\begin{array}{c}\text { Total } \\
\text { time } \\
(\mathbf{m i n})\end{array}$ & $\begin{array}{l}\text { Recorded cross-section } \\
\text { SELs }\end{array}$ & $\begin{array}{c}\left.\mathbf{c m}^{2} / \text { Device }\right) \\
\end{array}$ \\
\hline 60 & 16.6 & 24 & 15 & 296 & 0 & $1.83 \times 10^{-12 *}$ \\
155 & 10 & 18 & 20 & 50 & 0 & $7.98 \times 10^{-13 *}$ \\
210 & 11 & 15 & 24 & 110 & 0 & $2.35 \times 10^{-12 *}$ \\
\hline
\end{tabular}

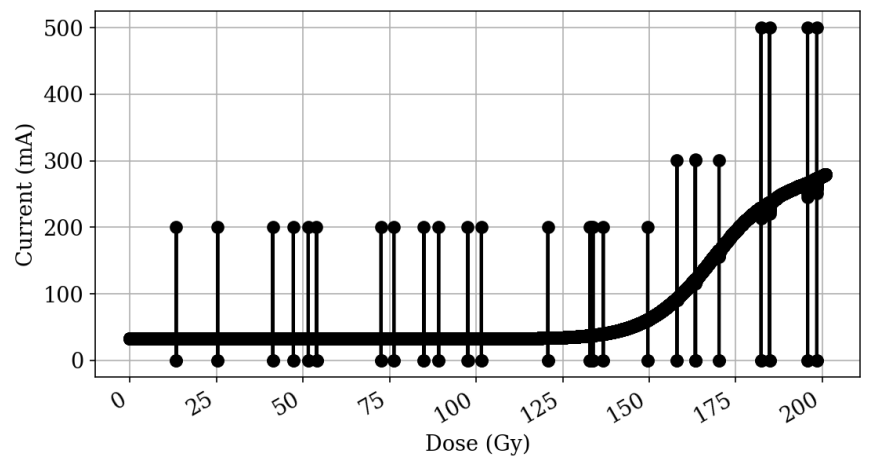

Fig. 2. Increase in current observed in the ISSI SRAM standby current during the experiments at VESPER. The current limit for testing is always $200 \mathrm{~mA}$. After the current rose above an acceptable value, the results are not considered for cross section calculations and the current limit was set higher to observe the increase in current.

given for the Alliance memory are, therefore, upper limit values. The experimental data can be seen in Table V.

\section{TID Effects Observed During SEL Testing}

Due to the low cross section of electron-induced SEL, the DUT received a large deposited dose during the irradiation. During the experiments, an increase in the current consumption was noted, as seen in Fig. 2. After the current increase, the DUT continued to experience the SEL events. However, to make sure that the increase in the current did not affect the SEL cross section results whenever the current increased above the nominal the test results were disregarded.

To better understand if the increase in the current was really caused by the deposited dose a total ionizing dose (TID) test at the Co-60 facility at ESTEC in the Netherlands was conducted. All the three devices were exposed to the gamma radiation and the current was monitored during the whole experiment. All the DUT were biased at $3.3 \mathrm{~V}$. For the Brilliance memory, the $C E$ and the $\overline{C E}$ pin were connected according to specification so that the memory was turned off.

The increase of current in the three devices can be seen in Fig. 3. The overall conclusions for the test were that the current consumption of the ISSI memory starts to increase after about $330 \mathrm{~Gy}$, of the Alliance memory after about $550 \mathrm{~Gy}$, and of the Brilliance memory after about $270 \mathrm{~Gy}$. Therefore, extra care should be taken when testing components for electroninduced SELs due to the large associated fluences to obtain 

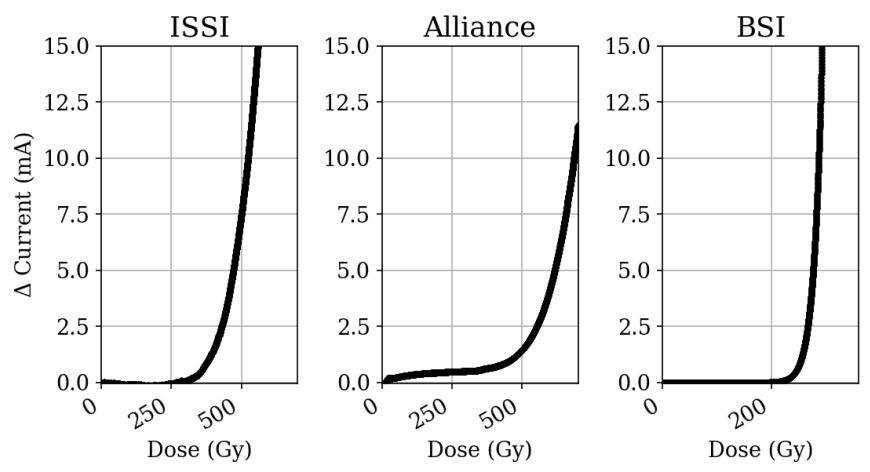

Fig. 3. Increase of the standby current in SRAM biased to nominal voltage. All the three devices showed an increase in current similar to the one observed at the VESPER facility. The change from standby current is shown for easier comparison.
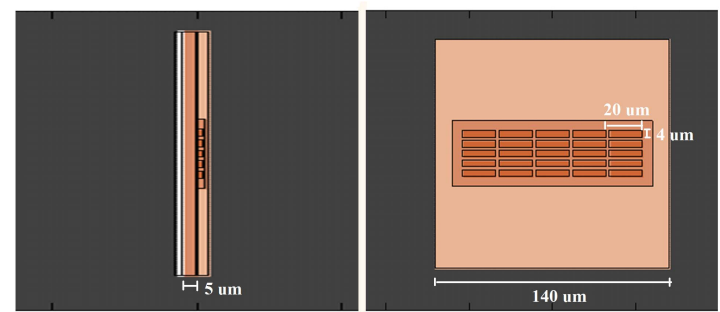

Fig. 4. Geometry of the SVs and BEOL.

statistically meaningful results and the possible associated TID impact.

\section{AnAlysis of the ELECTRON-INDUCED SEL MECHANISMS}

\section{A. FLUKA Energy Deposition Simulation}

To identify the effect that high- $Z$ materials have on the energy deposited by HE electrons, protons, shown previously in [2], and photons in the sensitive volume (SV) of the SRAM, a FLUKA simulation was performed using a geometry described in [13] and [14] where it is shown to be representative of the SRAMs used in this paper. The geometry used in this paper is verified for the same components [13] for protons; as both protons and neutrons cause latchup events through a similar mechanism-silicon fragments caused by nuclear reaction with the Si atoms. The geometry can be seen in Fig. 4. The ISSI and Alliance memories were represented with an SV thickness of 2.5 and $3.2 \mu \mathrm{m}$, respectively, in [12], however, for a first assessment the $2-\mu \mathrm{m} \mathrm{SV}$ thickness is found as a satisfactory approximation of the most accurate SV thickness.

The geometry used is an IRPP model in grid of $5 \times 5 \mathrm{SVs}$ with a size of $20 \mu \mathrm{m} \times 2 \mu \mathrm{m} \times 4 \mu \mathrm{m}$ and a back-end-of-line (BEOL) of $5 \mu \mathrm{m}$ of $\mathrm{SiO}_{2}$. In addition to this, a layer of 122-nm tungsten placed $200 \mathrm{~nm}$ above the SVs, to assess the effect that high-Z materials have on the energy deposition. It was stated in [13] that $5 \mu \mathrm{m}$ of $\mathrm{SiO}_{2}$ is a good estimation of the BEOL as the cross section at higher energies, also at $60 \mathrm{MeV}$, is dominated by silicon fragments and adding aluminum and/or $\mathrm{SiO}_{2}$ will not affect the final result.

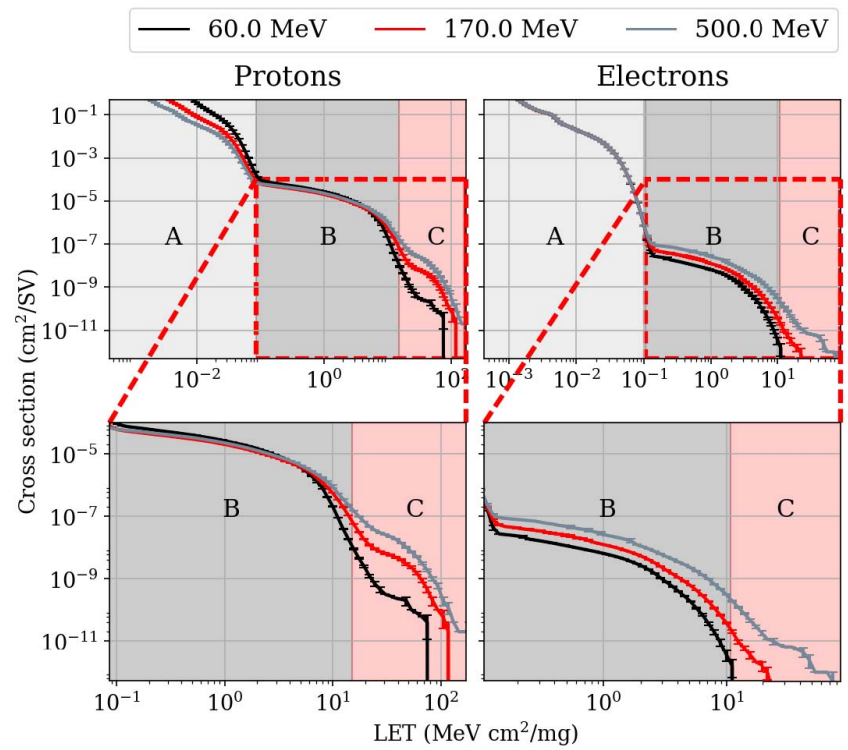

Fig. 5. Reverse integral of the energy deposition simulation in the SVs. Bottom row is a zoomed version of the top row to highlight the increase in cross section due to the tungsten recoils. (a) Direct ionization region. (b) Indirect ionization region and silicon recoils. (c) Tertiary region created by recoils from particle-tungsten interaction.

A beam of either electrons, protons, or photons impinged on the BEOL, thereafter, the energy deposited in each SV was scored. The resulting reverse integral cross section can be seen in Fig. 5. The cross section has been normalized by the simulated beam surface and the actual SV surface [12].

There are several things to note when comparing the proton and electron cross sections. (I) The electron and proton cross sections have a similar shape, with a direct ionization region (marked as A in Fig. 5) and a secondary indirect ionization region (marked as B in Fig. 5). This region is composed of secondary recoils that are created by both electron and proton interaction with silicon. (II) The proton indirectionization cross section has no significant energy dependence, whereas electron indirect-ionization cross section shows a strong energy dependence. Both points (I) and (II) have been studied in [6]. (III) The introduction of tungsten in the BEOL of the device in the simulation introduces the third region (marked as $\mathrm{C}$ in Fig. 5) in the cross section. This region is caused by fission fragments created by tungsten interaction with the particles, as shown in [2] for protons. The region is not as apparent for electrons due to the lower probability and lower LET of these recoils. As opposed to region (B), the energy deposition in the region $(\mathrm{C})$ range exhibits a strong dependence with the incidence particle energy.

\section{B. FLUKA Estimation of the Cross Sections}

To be able to estimate the cross section of the electroninduced SEL effects, an approach described in [12] has been used. First, the heavy-ion cross section of the memories is found experimentally and a Weibull function is fit to the experimental data to obtain the relevant quantities for further analysis. They are the LET threshold Lo, the saturated 




Fig. 6. Simulated SEL cross section for Alliance.



Fig. 7. Simulated SEL cross section for Brilliance.

cross section value XSsat, the width parameter $\mathrm{W}$ and the shape parameter s. Then, energy deposition simulations are performed in FLUKA. Thereafter, the Weibull curve and the energy deposition curve obtained in FLUKA are folded for each primary beam energy simulation from FLUKA. Through this operation, an estimation of the cross section for different particle types, in this case, electrons, is obtained. The procedure for the folding is outlined in detail in [13]. The Weibull parameters for the SRAM mentioned in this paper have been characterized previously in a heavy-ion beam [12], [13] and can be seen summarized in Table II.

The resulting estimated cross section for Alliance, Brilliance, and ISSI can be seen in Figs. 6, 7, and 8, respectively. Although the memories all have different Weibull characteristics, the IRPP model used in this paper should be a good estimate for the overall shape and cross section for the memories. The match is verified by applying the same method as used in [13] for protons and achieving a satisfactory match between the simulation and experimental data.

For the Brilliance memory, the fit with the simulated cross section follows the overall shape and absolute value of the cross section. The lower energy point also follows the theoretical decrease in cross section well.

The ISSI memory follows the overall shape of the estimated cross section curve well, however, the absolute value of the experimental points is about a factor 4 higher than the simulated cross section. This can be mainly attributed to the fact that the IRPP model chosen for this paper is very sensitive to the changes in the thickness of the SVs [12].

The Alliance result compared with the simulated cross section indicates that all the SEL upper limits are above the simulated cross section which would explain why no SEL results were observed. In addition, the high onset LET

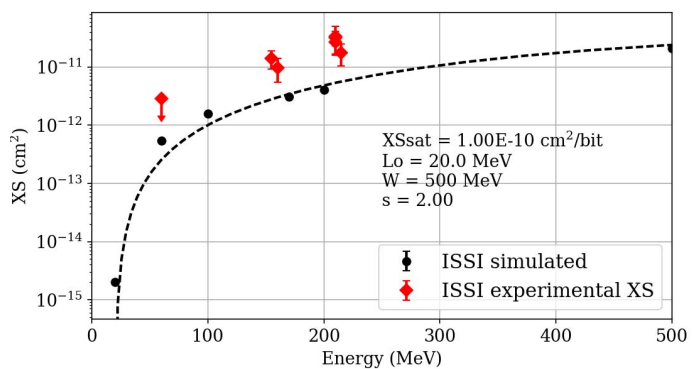

Fig. 8. Simulated SEL cross section for ISSI.

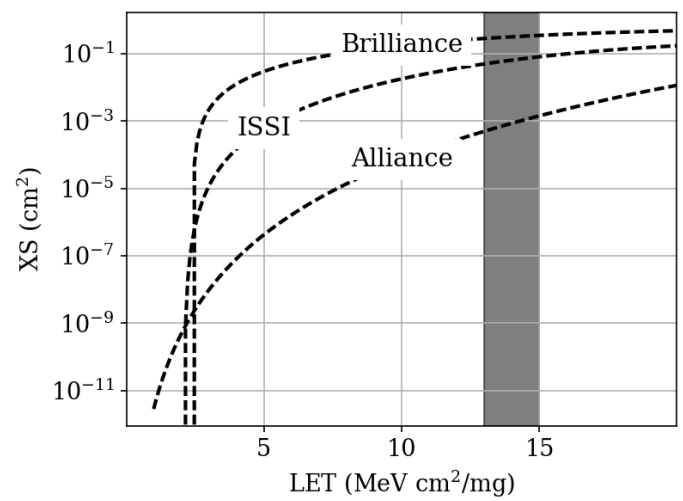

Fig. 9. Heavy-ion Weibull functions of the tested SRAM. Shaded area represents the highest LET of recoils generated by electrons and protons and illustrates the difference in the cross section in this region.

value of $15 \mathrm{MeV} \mathrm{cm} / 2 \mathrm{mg}$ explains why the cross section for this memory is lower compared to the other two memories. An additional limitation for reaching lower cross sections with the Alliance SRAM is the TID onset limit described in Section III-D. As an illustration, the Weibull functions for the memories together with the relevant LET range for electrons $(13 \mathrm{MeV} \mathrm{cm} / \mathrm{mg})$ and protons $\left(15 \mathrm{MeV} \mathrm{cm}^{2} / \mathrm{mg}\right)$ is plotted in Fig. 9. The heavy-ion cross section in this regions is about two magnitudes lower for the Alliance memory than for the ISSI and the Brilliance memories.

\section{FLUKA Tungsten Fragment Simulation}

It has been shown that heavy- $Z$ materials present in devices increase the SEL cross section during proton testing due to the high-LET recoils created by nuclear reactions [1]. To understand if electrons are similarly capable of producing fragments with LET values high enough to induce this effect, a tungsten nuclear fragment simulation was performed. A pencil beam of either protons, photons, or electrons impinged on a cube of $5-\mu \mathrm{m}$ side of tungsten. The quantities scored for the fragments are the LET in silicon, seen in Fig. 10, the charge, seen in Fig. 11 and the kinetic energy seen in Fig. 12. All these quantities were normalized to the inelastic interaction cross section, as seen in Table VI.

The secondary fragments created in tungsten by protons, as can be seen in Fig. 10, have LETs up to $\sim 45 \mathrm{MeVcm}^{2} / \mathrm{mg}$. These fragments are shown to be responsible for the increase of cross section in highly integrated circuits between 200 and $500 \mathrm{MeV}$ [2]. Fig. 10 also shows that the secondary 


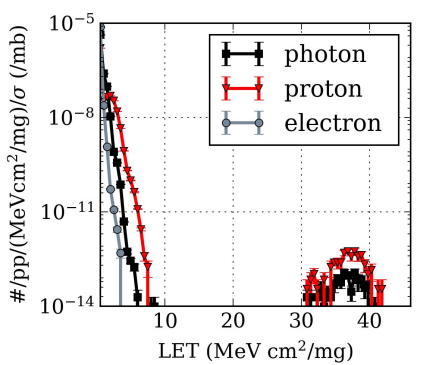

(a)



(b)
Fig. 10. Simulation of the LET of fragments generated by interaction with tungsten by protons, electrons, and photons. (a) $50-\mathrm{MeV}$ beam. (b) $200-\mathrm{MeV}$ beam.



(a)

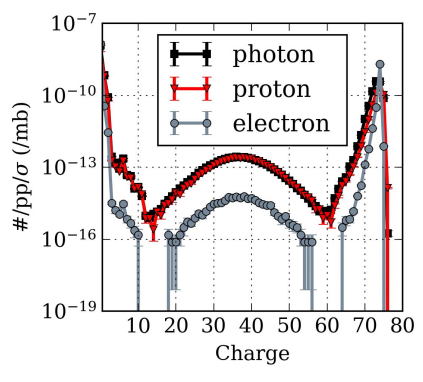

(b)
Fig. 11. Simulation of the atomic number of the fission fragments generated by interaction with tungsten by protons, electrons, and photons. (a) $50-\mathrm{MeV}$ beam. (b) $200-\mathrm{MeV}$ beam.

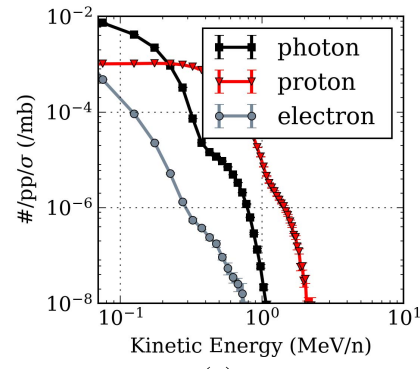

(a)

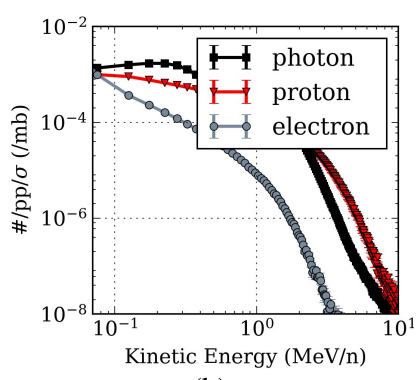

(b)
Fig. 12. Simulation of the kinetic energy of recoils generated by interaction with tungsten by protons, electrons, and photons. (a) $50-\mathrm{MeV}$ beam. (b) $200-\mathrm{MeV}$ beam.

fragments created by electrons in tungsten can have LETs up to $\sim 40 \mathrm{MeVcm}^{2} / \mathrm{mg}$. They are, therefore, theoretically capable to produce a similar increase in cross section. Moreover, the highest LET values of the fragments created by electrons in tungsten are very similar to that of the protons. In addition, the tungsten enhancement effect creates a strong energy dependence for the maximum LET that one can observe during electron testing. As seen in Fig. 10 on the left, at lower beam energies only siliconlike and tungstenlike secondary recoils are present.

\section{RHA IMPLICATIONS}

A possible strategy for assuring that components are free of proton-induced SEL is to first test with heavy-ions up to a given LET. For devices that do not include high- $Z$ material, this limit is set to $\sim 15 \mathrm{MeVcm}^{2} / \mathrm{mg}$. Due to the fact that high-Z materials interaction with protons creates high-LET
TABLE VI

Inelastic Interaction Cross Section for Electron, Photons, and Protons Used to Normalize the Results of the FluKA TUNGSTEN RECOIL SiMULATION

\begin{tabular}{cccc}
\hline $\begin{array}{c}\text { Energy } \\
(\mathbf{M e V})\end{array}$ & $\begin{array}{c}\text { Electron } \\
(\mathbf{m b})\end{array}$ & $\begin{array}{c}\text { Photon } \\
(\mathbf{m b})\end{array}$ & Proton $(\mathbf{m b})$ \\
\hline 50.0 & 0.325 & 2.1 & 578.0 \\
200 & 0.648 & 3.98 & 403.0 \\
\hline
\end{tabular}

fission fragments, it has been suggested that the heavy-ion test should be performed with LET of at least $40 \mathrm{MeV} \mathrm{cm}^{2} / \mathrm{mg}$ [2]. It was also suggested in [2] that to ensure that the component is completely SEL free for a given environment, one would have to test with the maximum energy of protons present in the environment. In this paper, it was shown that the photons and electrons are also capable of producing high-LET fragments of up to $40 \mathrm{MeV} \mathrm{cm}^{2} / \mathrm{mg}$, however, with a lower probability than protons. Therefore, in principle, a similar guideline is relevant for electrons. If the intended environment contains high-energetic electrons testing up to the aforementioned limit is also necessary in this case. The SEL cross section for the memories in this paper has an electron-induced SEL cross section which is several magnitudes lower than for the equivalent proton cross section. For the Brilliance memory, a proton saturation cross section has been found to be $\sim 3 \times 10^{-7} \mathrm{~cm}^{2}$ [12], whereas for electron testing it was found to be $3.3 \times 10^{-10} \mathrm{~cm}^{2}$. HE electron facilities still being relatively rare, they usually provide quite a low flux and energy of electrons. In addition, they are generally not built with electronics testing in mind. Therefore, SEL testing at these facilities can be time-consuming and quite complicated. Therefore, an alternative approach is to perform the tests with protons instead since the produced fragments have similar LETs. Another advantage is that testing can be done in less time due to the lower fluence. However, this is not the case for direct-ionization or elastic scattering, which have a higher cross section than the indirect ionization events. It was suggested in [2] that one has to consider the environment in which the device is to be operated in: the flux of the particles and the highest energy present in the environment, in which the device is to be used. The same consideration can be applied to electron testing, with the additional consideration that it is possible to substitute for proton testing instead. If the device is not sensitive to proton-induced SEL, it will not be sensitive to electron-induced SEL.

\section{CONCLUSION}

First experimental evidence showing electron-induced SEL has been shown in this paper. The DUTs had a LET threshold of $\sim 3 \mathrm{MeV} \mathrm{cm}^{2} / \mathrm{mg}$ [14]. It has been shown through simulation in [6] that electrons and photons are capable of producing nuclear interaction product with a LET of up to $13 \mathrm{MeV} \mathrm{cm}^{2} / \mathrm{mg}$ and $15 \mathrm{MeV} \mathrm{cm}^{2} / \mathrm{mg}$, respectively. Therefore, the SEL observed in the DUT are attributed to these products. Moreover, a device with a LET onset value of $15 \mathrm{MeV} \mathrm{cm}^{2} / \mathrm{mg}$ experienced no SEL events, which further implies that the recoils with high LETs are responsible for the SEL events. 
It has been shown in [2] that the presence of high-Z materials in devices enhances the SEL cross section for high energy protons. The fission fragments created by the proton and tungsten interaction can have a LET of up to $\sim 45 \mathrm{MeVcm}^{2} / \mathrm{mg}$. Similarly, it was shown in this paper that electrons and photons are capable of producing fragments with a similar LET of up to $\sim 45 \mathrm{MeVcm}^{2} / \mathrm{mg}$, however, with a much lower probability.

The experimental result comparison with the cross section estimate obtained through FLUKA energy deposition simulations shows a good fit of the experimental data and simulation. The absolute value and the energy dependence were clearly seen in the Brilliance memory, the energy dependence was also seen on the ISSI memory. In addition, the ISSI memory was tested at different beam energies and the cross section results were within the uncertainties from each other indicating no pile-up effect, which would imply an exponential increase in cross section with the energy [5].

Finally, as electrons and photons are shown to be capable of producing recoils with similar LET values, the implication to RHA is similar to the one suggested for protons in [2]: one should test with the highest energy present in the intended environment. In addition, where heavy- $\mathrm{Z}$ materials are present fission fragment with LETs of up to $45 \mathrm{MeV} \mathrm{cm} / \mathrm{mg}$ are created. Therefore, testing with heavy-ions with LET values of at least $40 \mathrm{MeVcm}^{2} / \mathrm{mg}$ is advised [2]. Electrons and photons have been shown to produce fission fragments with similar LETs of up to $\sim 40 \mathrm{MeV} \mathrm{cm}^{2} / \mathrm{mg}$. Therefore, a similar approach is advised for electrons in order to cover the highest possible LET fragments generated. Proton testing can be seen as a suitable substitute since electrons, photons, and protons all produce fission fragments with similar LET values, due to tungsten interactions and beam-silicon recoils. The added benefit from proton testing is the lower fluence, and therefore, time and cost, needed for device qualification.

\section{ACKNOWLEDGMENT}

The authors would like to thank M. Muschitiello and A. Costantino for their assistance with the Co60 testing at ESTEC.

\section{REFERENCES}

[1] J. R. Schwank et al., "Hardness assurance test guideline for qualifying devices for use in proton environments," IEEE Trans. Nucl. Sci., vol. 56 , no. 4, pp. 2171-2178, Aug. 2009.

[2] J. R. Schwank et al., "Effects of particle energy on protoninduced single-event latchup," IEEE Trans. Nucl. Sci., vol. 52, no. 6, pp. 2622-2629, Dec. 2005.

[3] M. A. Clemens et al., "The effect of high-Z materials on proton-induced charge collection," IEEE Trans. Nucl. Sci., vol. 57, no. 6, pp. 3212-3218, Dec. 2010.

[4] M. A. Clemens, "Energy deposition mechanisms for proton- and neutron-induced single event upsets in modern electronic devices," Ph.D. dissertation, Fac. Graduate School Vanderbilt, Vanderbilt Univ., Nashville, TN, USA, May 2012.

[5] M. J. Gadlage, A. H. Roach, A. R. Duncan, M. W. Savage, and M. J. Kay, "Electron-induced single-event upsets in 45-nm and 28-nm bulk CMOS SRAM-based FPGAs operating at nominal voltage," IEEE Trans. Nucl. Sci., vol. 62, no. 6, pp. 2717-2724, Dec. 2015.

[6] M. Tali et al., "High-energy electron-induced SEUs and Jovian environment impact," IEEE Trans. Nucl. Sci., vol. 64, no. 8, pp. 2016-2022, Aug. 2017. [Online]. Available: http://ieeexplore. ieee.org/document/7944600/

[7] M. P. King et al., "Electron-induced single-event upsets in static random access memory," IEEE Trans. Nucl. Sci., vol. 60, no. 6, pp. 4122-4129, Dec. 2013.

[8] JUICE Team, "JUICE environment specification," ESTEC/ESA, Noordwijk, The Netherlands, Tech. Rep., 2015.

[9] D. M. Hiemstra and E. W. Blackmore, "LET spectra of proton energy levels from 50 to $500 \mathrm{MeV}$ and their effectiveness for single event effects characterization of microelectronics," IEEE Trans. Nucl. Sci., vol. 50, no. 6, pp. 2245-2250, Dec. 2003.

[10] A. Ferrari, P. R. Sala, A. Fasso, and J. Ranft, FLUKA: A MultiParticle Transport Code, document INFN-TC-05-11, Oct. 2005, doi: 10.5170/CERN-2005-010.

[11] T. T. Böhlen et al., "The FLUKA code: Developments and challenges for high energy and medical applications," Nucl. Data Sheets, vol. 120, pp. 211-214, Jun. 2014.

[12] R. G. Alía et al., "SEL cross section energy dependence impact on the high energy accelerator failure rate," IEEE Trans. Nucl. Sci., vol. 61, no. 6, pp. 2936-2944, Oct. 2014.

[13] R. G. Alía et al., "Energy dependence of tungsten-dominated SEL cross sections," IEEE Trans. Nucl. Sci., vol. 61, no. 5, pp. 2718-2726, Oct. 2014.

[14] R. G. Alía et al., "Sub-LET threshold SEE cross section dependency with ion energy," IEEE Trans. Nucl. Sci., vol. 62, no. 6, pp. 2797-2806, Dec. 2015.

[15] R. Harboe-Sørensen et al., "The technology demonstration module onboard PROBA-II," IEEE Trans. Nucl. Sci., vol. 58, no. 3, pp. 1001-1007, Jun. 2011.

[16] E. Petersen, "Soft error results analysis and error rate prediction," in Proc. IEEE NSREC Short Course, Jul. 2008. 